\title{
A fractional order pine wilt disease model with Caputo-Fabrizio derivative
}

\author{
Muhammad Altaf Khan ${ }^{1 *}$, Saif Ullah², K.O. Okosun ${ }^{3}$ and Kamil Shah ${ }^{1}$
}

${ }^{*}$ Correspondence:

altafdir@gmail.com

${ }^{1}$ Department of Mathematics, City University of Science and

Information Technology, Peshawar, Pakistan

Full list of author information is

available at the end of the article

\begin{abstract}
A Caputo-Fabrizio type fractional order mathematical model for the dynamics of pine wilt disease (FPWD) is presented. The basic properties of the model are investigated. The existence and uniqueness of the solution for the proposed FPWD model are given via the fixed point theorem. The numerical simulations for the model are obtained by using particular parameter values. The non-integer order derivative provides more flexible and deeper information about the complexity of the dynamics of the proposed FPWD model than the integer order models established before.
\end{abstract}

Keywords: Caputo-Fabrizio (CF) fractional derivative; Pint wilt disease; Mathematical model; Fixed point theorem; Numerical simulation

\section{Introduction}

Mathematical models in epidemiology are used widely in order to understand better the dynamics of an infectious disease $[1,2]$. The application of the mathematical models is not limited to only human diseases, but they are also widely applied in other phenomena of biological sciences, such as ecology, forest, etc. In the human life, forest has an important role, therefore, it is necessary to ensure the safety strategies to protect it from being infected with diseases. The forest provides greenery to the environment and pleasant atmosphere for humans. The pine wilt disease (PWD) infects pine trees and is one of the main threats to the ecosystem and forest. The PWD is considered to be the most destructive disease which damages pine trees in a short period of time, that is, a year or sometimes in a few months. The initial symptoms of the PWD include discoloration of needles, which turn from yellow to green then to reddish brown. The main agent of the disease is small worms, known as pinewood nematode (bursaphelenchus xylophilus), causing tree decline [3]. As the trees begin to die, they are attacked by insects, known as sawyers, which are species that transfer the nematode to healthy trees, which is one of the causes of pine wilt disease [4-6]. Native to North America, the PW nematode was introduced while the first epidemic of the PWD was accursed in Japan in 1905 [7], and it has spread in southern China, Korea, Taiwan, and other regions of Europe since the early 1980s [4-6, 8]. The PWD has three main organisms: the gymnosperm host, the pine wood nematode, and the insect vector. At the stage of primary transmission, dauer juveniles (JIV stage) of bursaphelenchus xylophilus are carried vertically in the tracheae of their beetle host to young twigs of susceptible trees, where they enter through resin canals in wounds made during maturation feeding by the insect [8].

(c) The Author(s) 2018. This article is distributed under the terms of the Creative Commons Attribution 4.0 International License (http://creativecommons.org/licenses/by/4.0/), which permits unrestricted use, distribution, and reproduction in any medium, provided you give appropriate credit to the original author(s) and the source, provide a link to the Creative Commons license, and indicate if changes were made. 
Recently, some mathematical models have been presented to explore the dynamics of PWD consisting of a system of nonlinear differential equations. Lee and Kwang [9] explored the stability analysis of PWD and proposed some suitable controlling strategies for this disease. Khan et al. [10] introduced a model on PWD and its optimal control. A mathematical model with variable population and suggested optimal control was developed in [11]. Most recently, in [12] the dynamics of PWD with saturated incidence rate was explored. All of the above PWD models are restricted to classical integer order differential equations. In the present paper we consider a PWD model with saturated incidence rate in fractional environment using the CF derivative. First, we give an overview of recently published papers on fractional mathematical models using the CF derivative.

Fractional order models are more reliable and helpful in the real phenomena than the classical models due to hereditary properties and the description of memory [13, 14]. Also, in the real world explanation, the integer order derivative does not explore the dynamics between two different points. To deal with such failures of classical local differentiation, different concepts on differentiation with non-local or fractional orders have been developed in the existing literature. For instance, Riemann and Liouville introduced the concept of fractional orders differentiation in [14]. Recently, Caputo and Fabrizio [15] introduced a new derivative with fractional order based on the exponential kernel. The new CF fractional order derivative has been used successfully in modeling of various real phenomena. For example, a fractional Adams-Bashforth technique via the CF derivative was presented in [16]. A study of magnetohydrodynamic electroosmotic flow of Maxwell fluids with CF derivatives was carried out by Abdulh et al. [17]. In [18], the CF fractional derivative was used for numerical approach of the Fokker-Planck equation using Ritz approximation. A mathematical comparative analysis of RL and $R C$ electrical circuits using $A B$ and $C F$ fractional derivatives was recently done in [19]. Mustafa et al.[20] explored the dynamics of the cancer treatment model with the CF fractional derivative. Recently, a new fractional model of hepatitis B virus in the CF derivative sense was presented in [21].

The classical integer order mathematical model is useful for a local dynamic system with no external forces. These models cannot therefore replicate the complexity of the dynamics of the communicable disease like PWD as the model can sometimes have a crossover behavior and this cannot be handled by the classical differential operators. Further, in the literature fractional order models provide a better fit to the real data for different diseases and other experimental work in fluid mechanics. For example, Diethelm [22] provided a good agreement to the real data of the 2009 dengue outbreak in Cape Verde using a noninteger order biological model instead of the ordinary one. A fractional order model for Ebola epidemic was applied to provide a suitable approximation to the real data on Ebola virus [23]. Makris et al. [24] used a fractional order Maxwell model to attain a better fit to the experimental work.

Therefore, motivated by the above work, in this paper, we aim to extend the recently published PWD model [12] to a fractional case by using the newly established derivative known as $C F$ derivative of order $\tau \in(0,1]$. The details of the remaining sections of this paper are as follows: The basic definition and results of fractional order derivative are stated in Sect. 2. In Sect. 3, we explore the model formulation, model equilibria, and the basic reproduction number. Section 4 deals with the existence of solution in the spread PWD disease model via the fixed point theorem. Also, the uniqueness of a model solution is 
obtained. Numerical simulations are presented in Sect. 5. Finally, the concluding remarks are given in Sect. 6.

\section{Preliminaries}

Here, we give some basic definitions of the fractional calculus that will be used in the onward analysis of the model.

Definition 1 Let $g \in H^{1}(a, b)$, with $b$ greater than $a, \tau \in[0,1]$, then the CF fractional derivative [15] is given as

$$
D_{t}^{\tau}(g(t))=\frac{M(\tau)}{1-\tau} \int_{a}^{t} g^{\prime}(x) \exp \left[-\tau \frac{t-x}{1-\tau}\right] d x .
$$

In Eq. (1) $M(\tau)$ represents a normality with $M(0)=M(1)=1$ [15]. However, if $g \notin H^{1}(a, b)$, then the following expression of the derivative is obtained:

$$
D_{t}^{\tau}(g(t))=\frac{\tau M(\tau)}{1-\tau} \int_{a}^{t}(g(t)-g(x)) \exp \left[-\tau \frac{t-x}{1-\tau}\right] d x .
$$

Remark 1 If $\sigma=\frac{1-\tau}{\tau} \in[0, \infty), \tau=\frac{1}{1+\sigma} \in[0,1]$, then Eq. (2) gives the following form:

$$
D_{t}^{\tau}(g(t))=\frac{N(\sigma)}{\sigma} \int_{a}^{t} g^{\prime}(x) \exp \left[-\frac{t-x}{\sigma}\right] d x, \quad N(0)=N(\infty)=1 .
$$

Moreover,

$$
\lim _{\sigma \rightarrow 0} \frac{1}{\sigma} \exp \left[-\frac{t-x}{\sigma}\right]=\delta(x-t) .
$$

Nieto and Losada [25] give the following definition of the integral.

Definition 2 Let $0<\tau<1$, then the fractional integral of the function $g$ having order $\tau$ is given below.

$$
I_{t}^{\tau}(g(t))=\frac{2(1-\tau)}{(2-\tau) M(\tau)} g(t)+\frac{2 \tau}{(2-\tau) M(\tau)} \int_{0}^{t} g(s) d s, \quad t \geq 0 .
$$

Remark 2 From Definition 2, we have

$$
\frac{2(1-\tau)}{(2-\tau) M(\tau)}+\frac{2 \tau}{(2-\tau) M(\tau)}=1
$$

which implies $M(\tau)=\frac{2}{2-\tau}, 0<\tau<1$. In view of (6), a new Caputo derivative of order $0<$ $\tau<1$ is suggested by Nieto and Losada [25], given as follows:

$$
D_{t}^{\tau}(g(t))=\frac{1}{1-\tau} \int_{0}^{t} g^{\prime}(x) \exp \left[\tau \frac{t-x}{1-\tau}\right] d x .
$$

The CF derivative [15], given in the above definitions, has been recently used in the mathematical modeling of HBV [21], Maxwell fluid with slip effects [26], and diabetes model [27]. 


\section{Model formulation}

Here, in this section, we extend the PWD model [12] to fractional order using a CF derivative of order $\tau \in[0,1]$. The classical integer order PWD model is formulated by the following nonlinear system of differential equations:

$$
\begin{aligned}
\frac{d S_{H}}{d t} & =\Pi_{H}-\frac{K_{1} S_{H} I_{V}}{1+\theta_{1} I_{V}}-\frac{K_{2} \psi S_{H} I_{V}}{1+\theta_{1} I_{V}}-\gamma_{H} S_{H}, \\
\frac{d E_{H}}{d t} & =\frac{K_{1} S_{H} I_{V}}{1+\theta_{1} I_{V}}-\gamma_{H} E_{H}-\delta_{H} E_{H}, \\
\frac{d I_{H}}{d t} & =\frac{K_{2} \psi S_{H} I_{V}}{1+\theta_{1} I_{V}}+\delta_{H} E_{H}-\gamma_{H} I_{H}, \\
\frac{d S_{V}}{d t} & =\Pi_{V}-\frac{\beta_{1} S_{V} I_{H}}{1+\theta_{2} I_{H}}-\gamma_{V} S_{V}, \\
\frac{d E_{V}}{d t} & =\frac{\beta_{1} S_{V} I_{H}}{1+\theta_{2} I_{H}}-\gamma_{V} E_{V}-\delta_{V} E_{V}, \\
\frac{d I_{V}}{d t} & =\delta_{V} E_{V}-\gamma_{V} I_{V} .
\end{aligned}
$$

In the above model (8), the total host population (pine trees) is denoted by $N_{H}(t)$. It is subdivided into three classes: susceptible $S_{H}(t)$, exposed $E_{H}(t)$, and infected $I_{H}(t)$ pine trees. The total vector population (beetles) is further divided into three subclasses: susceptible vector $S_{V}(t)$, exposed vectors $E_{V}(t)$, and infected vector $I_{V}(t)$. The recruitment rates of pine trees and vector population are denoted by $\Pi_{H}$ and $\Pi_{V}$, respectively. The rate of contact between suspectable trees and infected vectors is $K_{1}$, while $K_{2}$ is the contact rate between suspectable trees and infected vectors when the nematode is transmitted by the infected vector at oviposition. The natural death rates of pine trees and vector population are denoted by parameters $\gamma_{H}$ and $\gamma_{H}$, respectively. The natural death rate of pine trees which are uninfected through beetles is denoted by parameter $\psi$. The constants of saturation are $\theta_{1}$ and $\theta_{2}$. The exposed pine trees join the infected class at the rate $\delta_{H}$ while the transfer rate of an exposed vector to become an infected vector is denoted by $\delta_{V}$. The parameter $\beta_{1}$ is the contact rate of a suspectable vector with infected pine trees. We reformulate the classical PWD model (8) by replacing the ordinary integer order derivative by the new $\mathrm{CF}$ fractional derivative and it can be written as follows:

$$
\begin{aligned}
& { }_{0}^{C F} D_{t}^{\tau} S_{H}=\Pi_{H}-\frac{K_{1} S_{H} I_{V}}{1+\theta_{1} I_{V}}-\frac{K_{2} \psi S_{H} I_{V}}{1+\theta_{1} I_{V}}-\gamma_{H} S_{H}, \\
& { }_{0}^{C F} D_{t}^{\tau} E_{H}=\frac{K_{1} S_{H} I_{V}}{1+\theta_{1} I_{V}}-\gamma_{H} E_{H}-\delta_{H} E_{H}, \\
& { }_{0}^{C F} D_{t}^{\tau} I_{H}=\frac{K_{2} \psi S_{H} I_{V}}{1+\theta_{1} I_{V}}+\delta_{H} E_{H}-\gamma_{H} I_{H}, \\
& { }_{0}^{C F} D_{t}^{\tau} S_{V}=\Pi_{V}-\frac{\beta_{1} S_{V} I_{H}}{1+\theta_{2} I_{H}}-\gamma_{V} S_{V}, \\
& { }_{0}^{C F} D_{t}^{\tau} E_{V}=\frac{\beta_{1} S_{V} I_{H}}{1+\theta_{2} I_{H}}-\gamma_{V} E_{V}-\delta_{V} E_{V}, \\
& { }_{0}^{C F} D_{t}^{\tau} I_{V}=\delta_{V} E_{V}-\gamma_{V} I_{V} .
\end{aligned}
$$


The initial conditions involved in (9) are

$$
\begin{aligned}
& S_{H}(0)=c_{1}, \quad E_{H}(0)=c_{2}, \quad I_{H}(0)=c_{3}, \quad S_{V}(0)=c_{4}, \\
& E_{V}(0)=c_{5} \quad \text { and } \quad I_{V}(0)=c_{6} .
\end{aligned}
$$

\subsection{Equilibria and basic reproduction number}

Model (9) has a disease free equilibrium $E^{0}=\left(S_{H}^{o}, 0,0, S_{V}^{o}, 0,0\right)$ and is obtained by solving the system

$$
{ }_{0}^{C F} D_{t}^{\tau} S_{H}={ }_{0}^{C F} D_{t}^{\tau} E_{H}={ }_{0}^{C F} D_{t}^{\tau} I_{H}={ }_{0}^{C F} D_{t}^{\tau} C={ }_{0}^{C F} D_{t}^{\tau} S_{V}={ }_{0}^{C F} D_{t}^{\tau} E_{V}={ }_{0}^{C F} D_{t}^{\tau} I_{V}=0,
$$

and is given by

$$
E^{0}=\left(\frac{\Pi_{H}}{\gamma_{H}}, 0,0, \frac{\Pi_{V}}{\gamma_{V}}, 0,0\right)
$$

The model has a unique endemic equilibrium, denoted by $E_{1}$, given by

$$
\left\{\begin{array}{l}
S_{H}^{*}=\frac{\Pi_{H}\left(1+\theta_{1} I_{V}^{*}\right)}{\gamma_{H}+I_{V}^{*}\left(K_{1}+K_{2} \psi+\gamma_{H} \theta_{1}\right)} \\
E_{H}^{*}=\frac{\Pi_{H} K_{1} I_{V}^{*}}{\left(\gamma_{H}+\delta_{H}\right)\left(K_{1} I_{V}^{*}+K_{2} \psi I_{V}^{*}+\gamma_{H}+\theta_{1} \gamma_{H} I_{V}^{*}\right)} \\
I_{H}^{*}=\frac{\Pi_{H} I_{V}^{*}\left(K_{2} \psi \gamma_{H}+K_{1} \delta_{H}+K_{2} \psi \delta_{H}\right)}{\gamma_{H}\left(\gamma_{H}+\delta_{H}\right)\left(K_{1} I_{V}^{*}+K_{2} I_{V}^{*}+\gamma_{H}+\theta_{1} \gamma_{H} I_{V}^{*}\right)} \\
S_{V}^{*}=\frac{\Pi_{V}\left(1+\theta_{2} I_{H}^{*}\right)}{\gamma_{V}+I_{H}^{*}\left(\beta_{1}+\theta_{2} \gamma_{V}\right)} \\
E_{V}^{*}=\frac{\Pi_{V} \beta_{1} I_{H}^{*}}{\left(\gamma_{V}+\delta_{V}\right)\left(\gamma_{V}+I_{H}^{*}\left(\beta_{1}+\theta_{2} \gamma_{V}\right)\right)} \\
I_{V}^{*}=\frac{\Pi_{V} \beta_{1} \delta_{V} I_{H}^{*}}{\gamma_{V}\left(\gamma_{V}+\delta_{V}\right)\left(\gamma_{V}+I_{H}^{*}\left(\beta_{1}+\theta_{2} \gamma_{V}\right)\right)}
\end{array}\right.
$$

The basic reproduction number $\mathcal{R}_{0}$ is obtained by using the next generation technique [28] and is given as follows:

$$
\mathcal{R}_{0}=\sqrt{\frac{\delta_{V} \beta_{1} S_{H}^{0} S_{V}^{0}\left(K_{1} \delta_{H}+K_{2} \psi\left(\gamma_{H}+\delta_{H}\right)\right)}{\gamma_{H} \gamma_{V}\left(\gamma_{H}+\delta_{H}\right)\left(\gamma_{V}+\delta_{V}\right)}} .
$$

Hence, we state the following theorem.

Theorem 1 The FPWD model (9) has a unique endemic equilibrium if $\mathcal{R}_{0}>1$.

\section{Existence and uniqueness of FPWD model}

This section describes the existence of model solutions by using fixed point theory. We use the fractional integral operator in [25] on (9) to obtain

$$
\begin{aligned}
& S_{H}(t)-S_{H}(0)={ }_{0}^{C F} I_{t}^{\tau}\left\{\Pi_{H}-\frac{K_{1} S_{H} I_{V}}{1+\theta_{1} I_{V}}-\frac{K_{2} \psi S_{H} I_{V}}{1+\theta_{1} I_{V}}-\gamma_{H} S_{H}\right\}, \\
& E_{H}(t)-E_{H}(0)={ }_{0}^{C F} I_{t}^{\tau}\left\{\frac{K_{1} S_{H} I_{V}}{1+\theta_{1} I_{V}}-\gamma_{H} E_{H}-\delta_{H} E_{H}\right\}, \\
& I_{H}(t)-I_{H}(0)={ }_{0}^{C F} I_{t}^{\tau}\left\{\frac{K_{2} \psi S_{H} I_{V}}{1+\theta_{1} I_{V}}+\delta_{H} E_{H}-\gamma_{H} I_{H}\right\}, \\
& S_{V}(t)-S_{V}(0)={ }_{0}^{C F} I_{t}^{\tau}\left\{\Pi_{V}-\frac{\beta_{1} S_{V} I_{H}}{1+\theta_{2} I_{H}}-\gamma_{V} S_{V}\right\},
\end{aligned}
$$




$$
\begin{aligned}
& E_{V}(t)-E_{V}(0)={ }_{0}^{C F} I_{t}^{\tau}\left\{\frac{\beta_{1} S_{V} I_{H}}{1+\theta_{2} I_{H}}-\gamma_{V} E_{V}-\delta_{V} E_{V}\right\}, \\
& I_{V}(t)-I_{V}(0)={ }_{0}^{C F} I_{t}^{\tau}\left\{\delta_{V} E_{V}-\gamma_{V} I_{V}\right\} .
\end{aligned}
$$

Applying the idea used in [25], we obtain

$$
\begin{aligned}
& S_{H}(t)-S_{H}(0)=\frac{2(1-\tau)}{(2-\tau) M(\tau)}\left\{\Pi_{H}-\frac{K_{1} S_{H} I_{V}}{1+\theta_{1} I_{V}}-\frac{K_{2} \psi S_{H} I_{V}}{1+\theta_{1} I_{V}}-\gamma_{H} S_{H}\right\} \\
& +\frac{2 \tau}{(2-\tau) M(\tau)} \int_{0}^{t}\left\{\Pi_{H}-\frac{K_{1} S_{H} I_{V}}{1+\theta_{1} I_{V}}-\frac{K_{2} \psi S_{H} I_{V}}{1+\theta_{1} I_{V}}-\gamma_{H} S_{H}\right\} d y, \\
& E_{H}(t)-E_{H}(0)=\frac{2(1-\tau)}{(2-\tau) M(\tau)}\left\{\frac{K_{1} S_{H} I_{V}}{1+\theta_{1} I_{V}}-\gamma_{H} E_{H}-\delta_{H} E_{H}\right\} \\
& +\frac{2 \tau}{(2-\tau) M(\tau)} \int_{0}^{t}\left\{\frac{K_{1} S_{H} I_{V}}{1+\theta_{1} I_{V}}-\gamma_{H} E_{H}-\delta_{H} E_{H}\right\} d y, \\
& I_{H}(t)-I_{H}(0)=\frac{2(1-\tau)}{(2-\tau) M(\tau)}\left\{\frac{K_{2} \psi S_{H} I_{V}}{1+\theta_{1} I_{V}}+\delta_{H} E_{H}-\gamma_{H} I_{H}\right\} \\
& +\frac{2 \tau}{(2-\tau) M(\tau)} \int_{0}^{t}\left\{\frac{K_{2} \psi S_{H} I_{V}}{1+\theta_{1} I_{V}}+\delta_{H} E_{H}-\gamma_{H} I_{H}\right\} d y, \\
& S_{V}(t)-S_{V}(0)=\frac{2(1-\tau)}{(2-\tau) M(\tau)}\left\{\Pi_{V}-\frac{\beta_{1} S_{V} I_{H}}{1+\theta_{2} I_{H}}-\gamma_{V} S_{V}\right\} \\
& +\frac{2 \tau}{(2-\tau) M(\tau)} \int_{0}^{t}\left\{\Pi_{V}-\frac{\beta_{1} S_{V} I_{H}}{1+\theta_{2} I_{H}}-\gamma_{V} S_{V}\right\} d y, \\
& E_{V}(t)-E_{V}(0)=\frac{2(1-\tau)}{(2-\tau) M(\tau)}\left\{\frac{\beta_{1} S_{V} I_{H}}{1+\theta_{2} I_{H}}-\gamma_{V} E_{V}-\delta_{V} E_{V}\right\} \\
& +\frac{2 \tau}{(2-\tau) M(\tau)} \int_{0}^{t}\left\{\frac{\beta_{1} S_{V} I_{H}}{1+\theta_{2} I_{H}}-\gamma_{V} E_{V}-\delta_{V} E_{V}\right\} \\
& I_{V}(t)-I_{V}(0)=\frac{2(1-\tau)}{(2-\tau) M(\tau)}\left\{\delta_{V} E_{V}-\gamma_{V} I_{V}\right\} \\
& +\frac{2 \tau}{(2-\tau) M(\tau)} \int_{0}^{t}\left\{\delta_{V} E_{V}-\gamma_{V} I_{V}\right\} d y .
\end{aligned}
$$

For simplicity, we replace as follows:

$$
\begin{aligned}
& \digamma_{1}\left(t, S_{H}\right)=\Pi_{H}-\frac{K_{1} S_{H} I_{V}}{1+\theta_{1} I_{V}}-\frac{K_{2} \psi S_{H} I_{V}}{1+\theta_{1} I_{V}}-\gamma_{H} S_{H}, \\
& \digamma_{2}\left(t, E_{H}\right)=\frac{K_{1} S_{H} I_{V}}{1+\theta_{1} I_{V}}-\gamma_{H} E_{H}-\delta_{H} E_{H}, \\
& \digamma_{3}\left(t, I_{H}\right)=\frac{K_{2} \psi S_{H} I_{V}}{1+\theta_{1} I_{V}}+\delta_{H} E_{H}-\gamma_{H} I_{H} \\
& \digamma_{4}\left(t, S_{V}\right)=\Pi_{V}-\frac{\beta_{1} S_{V} I_{H}}{1+\theta_{2} I_{H}}-\gamma_{V} S_{V} \\
& \digamma_{5}\left(t, E_{V}\right)=\frac{\beta_{1} S_{V} I_{H}}{1+\theta_{2} I_{H}}-\gamma_{V} E_{V}-\delta_{V} E_{V} \\
& \digamma_{6}\left(t, I_{V}\right)=\delta_{V} E_{V}-\gamma_{V} I_{V} .
\end{aligned}
$$


Theorem 2 The kernels $\digamma_{1}, \digamma_{2}, \digamma_{3}, \digamma_{4}, \digamma_{5}$, and $\digamma_{6}$ fulfill the Lipschitz condition and contraction if the following inequality holds:

$$
0 \leq\left(K_{1}+K_{2} \psi\right) e+\gamma_{H}<1
$$

Proof Here, we start from $\digamma_{1}$. Suppose $S$ and $S_{1}$ are two functions, then we assess the following:

$$
\begin{aligned}
\left\|\digamma_{1}\left(t, S_{H}\right)-\digamma_{1}\left(t, S_{1 H}\right)\right\| \\
=\|-\frac{K_{1} I_{V}}{1+\theta_{1} I_{V}}\left\{S_{H}(t)-S_{H}\left(t_{1}\right)\right\}-\frac{K_{2} \psi I_{V}}{1+\theta_{1} I_{V}}\left\{S_{H}(t)-S_{H}\left(t_{1}\right)\right\} \\
\quad-\gamma_{H}\left\{S_{H}(t)-S_{H}\left(t_{1}\right)\right\} \| .
\end{aligned}
$$

Using the triangular inequality on Eq. (13), we obtain

$$
\begin{aligned}
&\left\|\digamma_{1}\left(t, S_{H}\right)-\digamma_{1}\left(t, S_{1 H}\right)\right\| \\
& \leq\left\|\frac{K_{1} I_{V}}{1+\theta_{1} I_{V}}\left\{S_{H}(t)-S_{H}\left(t_{1}\right)\right\}\right\|+\left\|\frac{K_{2} \psi I_{V}}{1+\theta_{1} I_{V}}\left\{S_{H}(t)-S_{H}\left(t_{1}\right)\right\}\right\| \\
&+\gamma_{H}\left\|\left\{S_{H}(t)-S_{H}\left(t_{1}\right)\right\}\right\| \\
& \leq\left\{\frac{K_{1}}{1+\theta_{1} I_{V}}\left\|I_{V}(t)\right\|+\frac{K_{2} \psi}{1+\theta_{1} I_{V}}\left\|I_{V}(t)\right\|+\gamma_{H}\right\}\left\|\left\{S_{H}(t)-S_{H}\left(t_{1}\right)\right\}\right\| \\
& \leq\left.\left(K_{1}+K_{2} \psi\right)\left\|I_{V}\right\|+\gamma_{H}\right)\left\|\left\{S_{H}(t)-S_{H}\left(t_{1}\right)\right\}\right\| \\
& \leq\left(\left(K_{1}+K_{2} \psi\right) e+\gamma_{H}\right)\left\|\left\{S_{H}(t)-S_{H}\left(t_{1}\right)\right\}\right\| \\
& \leq \mu_{1}\left\|\left\{S(t)-S\left(t_{1}\right)\right\}\right\| .
\end{aligned}
$$

Taking $\mu_{1}=\left(K_{1}+K_{2} \psi\right) e+\gamma_{H}$, where $\left\|I_{H}(t)\right\| \leq e$ is a bounded function, we get

$$
\left\|\digamma_{1}\left(t, S_{H}\right)-\digamma_{1}\left(t, S_{1 H}\right)\right\| \leq \mu_{1}\left\|S_{H}(t)-S_{H}\left(t_{1}\right)\right\| .
$$

Hence, the Lipschitz condition is fulfilled for $\digamma_{1}$, and if in addition $0 \leq\left(K_{1}+K_{2} \psi\right) e+\gamma_{H}<$ 1 , then it is also a contraction. For the remaining cases, in a similar way the Lipschitz conditions are given as follows:

$$
\begin{aligned}
& \left\|\digamma_{2}\left(t, E_{H}\right)-\digamma_{2}\left(t, E_{1 H}\right)\right\| \leq \mu_{2}\left\|E_{H}(t)-E_{H}\left(t_{1}\right)\right\|, \\
& \left\|\digamma_{3}\left(t, I_{H}\right)-\digamma_{3}\left(t, I_{1 H}\right)\right\| \leq \mu_{3}\left\|I_{H}(t)-I_{H}\left(t_{1}\right)\right\|, \\
& \left\|\digamma_{4}\left(t, S_{V}\right)-\digamma_{4}\left(t, S_{1 V}\right)\right\| \leq \mu_{4}\left\|S_{V}(t)-S_{V}\left(t_{1}\right)\right\|, \\
& \left\|\digamma_{5}\left(t, E_{V}\right)-\digamma_{5}\left(t, E_{1 V}\right)\right\| \leq \mu_{5}\left\|E_{V}(t)-E_{V}\left(t_{1}\right)\right\|, \\
& \left\|\digamma_{6}\left(t, I_{V}\right)-\digamma_{6}\left(t, I_{1 V}\right)\right\| \leq \mu_{6}\left\|I_{V}(t)-I_{V}\left(t_{1}\right)\right\| .
\end{aligned}
$$


Using notations for kernels, Eq. (11) becomes

$$
\begin{aligned}
& S_{H}(t)=S_{H}(0)+\frac{2(1-\tau)}{(2-\tau) M(\tau)} \digamma_{1}\left(t, S_{H}\right)+\frac{2 \tau}{(2-\tau) M(\tau)} \int_{0}^{t}\left(\digamma_{1}\left(y, S_{H}\right)\right) d y, \\
& E_{H}(t)=E_{H}(0)+\frac{2(1-\tau)}{(2-\tau) M(\tau)} \digamma_{2}\left(t, E_{H}\right)+\frac{2 \tau}{(2-\tau) M(\tau)} \int_{0}^{t}\left(\digamma_{2}\left(y, E_{H}\right)\right) d y, \\
& I_{H}(t)=I_{H}(0)+\frac{2(1-\tau)}{(2-\tau) M(\tau)} \digamma_{3}\left(t, I_{H}\right)+\frac{2 \tau}{(2-\tau) M(\tau)} \int_{0}^{t}\left(\digamma_{3}\left(y, I_{H}\right)\right) d y, \\
& S_{V}(t)=S_{V}(0)+\frac{2(1-\tau)}{(2-\tau) M(\tau)} \digamma_{4}\left(t, S_{V}\right)+\frac{2 \tau}{(2-\tau) M(\tau)} \int_{0}^{t}\left(\digamma_{4}\left(y, S_{V}\right)\right) d y, \\
& E_{V}(t)=E_{V}(0)+\frac{2(1-\tau)}{(2-\tau) M(\tau)} \digamma_{5}\left(t, E_{V}\right)+\frac{2 \tau}{(2-\tau) M(\tau)} \int_{0}^{t}\left(\digamma_{5}\left(y, E_{V}\right)\right) d y, \\
& I_{V}(t)=I_{V}(0)+\frac{2(1-\tau)}{(2-\tau) M(\tau)} \digamma_{6}\left(t, I_{V}\right)+\frac{2 \tau}{(2-\tau) M(\tau)} \int_{0}^{t}\left(\digamma_{6}\left(y, I_{V}\right)\right) d y .
\end{aligned}
$$

The following recursive formula is presented:

$$
\begin{aligned}
& S_{H n}(t)=\frac{2(1-\tau)}{(2-\tau) M(\tau)} \digamma_{1}\left(t, S_{H(n-1)}\right)+\frac{2 \tau}{(2-\tau) M(\tau)} \int_{0}^{t}\left(\digamma_{1}\left(y, S_{H(n-1)}\right)\right) d y, \\
& E_{H n}(t)=\frac{2(1-\tau)}{(2-\tau) M(\tau)} \digamma_{2}\left(t, E_{H(n-1)}\right)+\frac{2 \tau}{(2-\tau) M(\tau)} \int_{0}^{t}\left(\digamma_{2}\left(y, E_{H(n-1)}\right)\right) d y, \\
& I_{H n}(t)=\frac{2(1-\tau)}{(2-\tau) M(\tau)} \digamma_{3}\left(t, I_{H(n-1)}\right)+\frac{2 \tau}{(2-\tau) M(\tau)} \int_{0}^{t}\left(\digamma_{3}\left(y, I_{H(n-1)}\right)\right) d y, \\
& S_{V n}(t)=\frac{2(1-\tau)}{(2-\tau) M(\tau)} \digamma_{4}\left(t, S_{V(n-1)}\right)+\frac{2 \tau}{(2-\tau) M(\tau)} \int_{0}^{t}\left(\digamma_{4}\left(y, S_{V(n-1)}\right)\right) d y, \\
& E_{V n}(t)=\frac{2(1-\tau)}{(2-\tau) M(\tau)} \digamma_{5}\left(t, E_{V(n-1)}\right)+\frac{2 \tau}{(2-\tau) M(\tau)} \int_{0}^{t}\left(\digamma_{5}\left(y, E_{V(n-1)}\right)\right) d y, \\
& I_{V n}(t)=\frac{2(1-\tau)}{(2-\tau) M(\tau)} \digamma_{6}\left(t, I_{V(n-1)}\right)+\frac{2 \tau}{(2-\tau) M(\tau)} \int_{0}^{t}\left(\digamma_{6}\left(y, I_{V(n-1)}\right)\right) d y
\end{aligned}
$$

with the initial conditions given below

$$
\left\{\begin{array}{l}
S_{H}^{0}(t)=S_{H}(0), \\
E_{H}^{0}(t)=E_{H}(0), \\
I_{H}^{0}(t)=I_{H}(0), \\
S_{V}^{0}(t)=S_{V}(0), \\
E_{V}^{0}(t)=E_{V}(0), \\
I_{V}^{0}(t)=I_{V}(0) .
\end{array}\right.
$$

The difference of the successive terms is calculated as follows:

$$
\begin{aligned}
\omega_{1 n}(t) & =S_{H n}(t)-S_{H(n-1)}(t) \\
& =\frac{2(1-\tau)}{(2-\tau) M(\tau)}\left(\digamma_{1}\left(t, S_{H(n-1)}\right)-\digamma_{1}\left(t, S_{H(n-2)}\right)\right)
\end{aligned}
$$




$$
\begin{aligned}
& +\frac{2 \tau}{(2-\tau) M(\tau)} \int_{0}^{t}\left(\digamma_{1}\left(y, S_{H(n-1)}\right)-\digamma_{1}\left(y, S_{H(n-2)}\right)\right) d y, \\
& \omega_{2 n}(t)=E_{H n}(t)-E_{H(n-1)}(t) \\
& =\frac{2(1-\tau)}{(2-\tau) M(\tau)}\left(\digamma_{2}\left(t, S_{H(n-1)}\right)-\digamma_{2}\left(t, S_{H(n-2)}\right)\right) \\
& +\frac{2 \tau}{(2-\tau) M(\tau)} \int_{0}^{t}\left(\digamma_{2}\left(y, E_{H(n-1)}\right)-\digamma_{2}\left(y, E_{H(n-2)}\right)\right) d y, \\
& \omega_{3 n}(t)=I_{H n}(t)-I_{H(n-1)}(t) \\
& =\frac{2(1-\tau)}{(2-\tau) M(\tau)}\left(\digamma_{3}\left(t, I_{H(n-1)}\right)-\digamma_{3}\left(t, I_{H(n-2)}\right)\right) \\
& +\frac{2 \tau}{(2-\tau) M(\tau)} \int_{0}^{t}\left(\digamma_{3}\left(y, I_{H(n-1)}\right)-\digamma_{3}\left(y, I_{H(n-2)}\right)\right) d y, \\
& \omega_{4 n}(t)=S_{V n}(t)-S_{V(n-1)}(t) \\
& =\frac{2(1-\tau)}{(2-\tau) M(\tau)}\left(\digamma_{4}\left(t, S_{V(n-1)}\right)-\digamma_{4}\left(t, S_{V(n-2)}\right)\right) \\
& +\frac{2 \tau}{(2-\tau) M(\tau)} \int_{0}^{t}\left(\digamma_{4}\left(y, S_{V(n-1)}\right)-\digamma_{4}\left(y, S_{V(n-2)}\right)\right) d y, \\
& \omega_{5 n}(t)=E_{V n}(t)-E_{V(n-1)}(t) \\
& =\frac{2(1-\tau)}{(2-\tau) M(\tau)}\left(\digamma_{5}\left(t, E_{V(n-1)}\right)-\digamma_{5}\left(t, E_{V(n-2)}\right)\right) \\
& +\frac{2 \tau}{(2-\tau) M(\tau)} \int_{0}^{t}\left(\digamma_{5}\left(y, E_{V(n-1)}\right)-\digamma_{5}\left(y, E_{V(n-2)}\right)\right) d y, \\
& \omega_{6 n}(t)=I_{V n}(t)-I_{V(n-1)}(t) \\
& =\frac{2(1-\tau)}{(2-\tau) M(\tau)}\left(\digamma_{6}\left(t, I_{V(n-1)}\right)-\digamma_{6}\left(t, I_{V(n-2)}\right)\right) \\
& +\frac{2 \tau}{(2-\tau) M(\tau)} \int_{0}^{t}\left(\digamma_{6}\left(y, I_{V(n-1)}\right)-\digamma_{6}\left(y, I_{V(n-2)}\right)\right) d y .
\end{aligned}
$$

Notice that

$$
\left\{\begin{array}{l}
S_{H n}(t)=\sum_{i=1}^{n} \omega_{1 i}(t), \\
E_{H n}(t)=\sum_{i=1}^{n} \omega_{2 i}(t), \\
I_{H n}(t)=\sum_{i=1}^{n} \omega_{3 i}(t), \\
S_{V n}(t)=\sum_{i=1}^{n} \omega_{4 i}(t), \\
E_{V n}(t)=\sum_{i=1}^{n} \omega_{5 i}(t), \\
I_{V n}(t)=\sum_{i=1}^{n} \omega_{6 i}(t) .
\end{array}\right.
$$

On continuing the same process, we assess

$$
\begin{aligned}
\left\|\omega_{1 n}(t)\right\| & =\left\|S_{H n}(t)-S_{H(n-1)}(t)\right\| \\
& =\| \frac{2(1-\tau)}{(2-\tau) M(\tau)}\left(\digamma_{1}\left(t, S_{H(n-1)}\right)-\digamma_{1}\left(t, S_{H(n-2)}\right)\right)
\end{aligned}
$$




$$
+\frac{2 \tau}{(2-\tau) M(\tau)} \int_{0}^{t}\left(\digamma_{1}\left(y, S_{H(n-1)}\right)-\digamma_{1}\left(y, S_{H(n-2)}\right)\right) d y \|
$$

Using the triangular inequality, Eq. (22) is simplified to

$$
\begin{aligned}
\left\|S_{H n}(t)-S_{H(n-1)}(t)\right\| \leq & \frac{2(1-\tau)}{(2-\tau) M(\tau)}\left\|\left(\digamma_{1}\left(t, S_{H(n-1)}\right)-\digamma_{1}\left(t, S_{H(n-2)}\right)\right)\right\| \\
& +\frac{2 \tau}{(2-\tau) M(\tau)}\left\|\int_{0}^{t}\left(\digamma_{1}\left(y, S_{H(n-1)}\right)-\digamma_{1}\left(y, S_{H(n-2)}\right)\right) d y\right\| .
\end{aligned}
$$

As the kernel fulfills the Lipschitz condition, then we have

$$
\begin{aligned}
\left\|S_{n}(t)-S_{n-1}(t)\right\| \leq & \frac{2(1-\tau)}{(2-\tau) M(\tau)} \mu_{1}\left\|S_{H(n-1)}-S_{H(n-2)}\right\|+\frac{2 \tau}{(2-\tau) M(\tau)} \mu_{1} \\
& \times \int_{0}^{t}\left\|S_{H(n-1)}-S_{H(n-2)}\right\| d y .
\end{aligned}
$$

Then we have

$$
\left\|\omega_{1 n}(t)\right\| \leq \frac{2(1-\tau)}{(2-\tau) M(\tau)} \mu_{1}\left\|\omega_{1(n-1)}(t)\right\|+\frac{2 \tau}{(2-\tau) M(\tau)} \mu_{1} \int_{0}^{t}\left\|\omega_{1(n-1)}(y)\right\| d y .
$$

Similarly, we get the following results:

$$
\begin{aligned}
\left\|\omega_{2 n}(t)\right\| & \leq \frac{2(1-\tau)}{(2-\tau) M(\tau)} \mu_{2}\left\|\omega_{2(n-1)}(t)\right\|+\frac{2 \tau}{(2-\tau) M(\tau)} \mu_{2} \int_{0}^{t}\left\|\omega_{2(n-1)}(y)\right\| d y, \\
\left\|\omega_{3 n}(t)\right\| & \leq \frac{2(1-\tau)}{(2-\tau) M(\tau)} \mu_{3}\left\|\omega_{3(n-1)}(t)\right\|+\frac{2 \tau}{(2-\tau) M(\tau)} \mu_{1} \int_{0}^{t}\left\|\omega_{3(n-1)}(y)\right\| d y, \\
\left\|\omega_{4 n}(t)\right\| & \leq \frac{2(1-\tau)}{(2-\tau) M(\tau)} \mu_{4}\left\|\omega_{4(n-1)}(t)\right\|+\frac{2 \tau}{(2-\tau) M(\tau)} \mu_{4} \int_{0}^{t}\left\|\omega_{4(n-1)}(y)\right\| d y, \\
\left\|\omega_{5 n}(t)\right\| & \leq \frac{2(1-\tau)}{(2-\tau) M(\tau)} \mu_{5}\left\|\omega_{5(n-1)}(t)\right\|+\frac{2 \tau}{(2-\tau) M(\tau)} \mu_{5} \int_{0}^{t}\left\|\omega_{5(n-1)}(y)\right\| d y, \\
\left\|\omega_{6 n}(t)\right\| & \leq \frac{2(1-\tau)}{(2-\tau) M(\tau)} \mu_{6}\left\|\omega_{6(n-1)}(t)\right\|+\frac{2 \tau}{(2-\tau) M(\tau)} \mu_{6} \int_{0}^{t}\left\|\omega_{6(n-1)}(y)\right\| d y .
\end{aligned}
$$

Now we state the theorem below.

Theorem 3 The FPWD model (9) has exact coupled solutions if the conditions below hold. That is, we can find $t_{0}$ such that

$$
\frac{2(1-\tau)}{(2-\tau) M(\tau)} \mu_{1}+\frac{2 \tau}{(2-\tau) M(\tau)} \mu_{1} t_{0}<1
$$

Proof Since all the functions $S_{H}(t), E_{H}(t), I_{H}(t)$ and $S_{V}(t), E_{V}(t), I_{V}(t)$ are bounded, we have shown that the kernels fulfill the Lipschitz condition, thus on using of Eqs. (25) and 
(26) and by using the recursive method, we obtain the succeeding relation as follows:

$$
\begin{aligned}
& \left\|\omega_{1 n}(t)\right\| \leq\left\|S_{H n}(0)\right\|\left[\left(\frac{2(1-\tau)}{(2-\tau) M(\tau)} \mu_{1}\right)+\left(\frac{2 \tau}{(2-\tau) M(\tau)} \mu_{1} t\right)\right]^{n}, \\
& \left\|\omega_{2 n}(t)\right\| \leq\left\|E_{H n}(0)\right\|\left[\left(\frac{2(1-\tau)}{(2-\tau) M(\tau)} \mu_{2}\right)+\left(\frac{2 \tau}{(2-\tau) M(\tau)} \mu_{2} t\right)\right]^{n}, \\
& \left\|\omega_{3 n}(t)\right\| \leq\left\|I_{H n}(0)\right\|\left[\left(\frac{2(1-\tau)}{(2-\tau) M(\tau)} \mu_{3}\right)+\left(\frac{2 \tau}{(2-\tau) M(\tau)} \mu_{3} t\right)\right]^{n}, \\
& \left\|\omega_{4 n}(t)\right\| \leq\left\|S_{V n}(0)\right\|\left[\left(\frac{2(1-\tau)}{(2-\tau) M(\tau)} \mu_{4}\right)+\left(\frac{2 \tau}{(2-\tau) M(\tau)} \mu_{4} t\right)\right]^{n}, \\
& \left\|\omega_{5 n}(t)\right\| \leq\left\|E_{V n}(0)\right\|\left[\left(\frac{2(1-\tau)}{(2-\tau) M(\tau)} \mu_{5}\right)+\left(\frac{2 \tau}{(2-\tau) M(\tau)} \mu_{5} t\right)\right]^{n}, \\
& \left\|\omega_{6 n}(t)\right\| \leq\left\|I_{V n}(0)\right\|\left[\left(\frac{2(1-\tau)}{(2-\tau) M(\tau)} \mu_{6}\right)+\left(\frac{2 \tau}{(2-\tau) M(\tau)} \mu_{6} t\right)\right]^{n} .
\end{aligned}
$$

Hence, the existence and continuity of the said solutions is proved. Furthermore, to ensure that the above function is a solution of Eq. (9), we proceed as follows:

$$
\begin{aligned}
& S_{H}(t)-S_{H}(0)=S_{H n}(t)-B_{n}(t), \\
& E_{H}(t)-E_{H}(0)=E_{H n}(t)-C_{n}(t), \\
& I_{H}(t)-I_{H}(0)=I_{H n}(t)-D_{n}(t), \\
& S_{V}(t)-S_{V}(0)=S_{V n}(t)-F_{n}(t), \\
& E_{V}(t)-E_{V}(0)=E_{V n}(t)-G_{n}(t), \\
& I_{V}(t)-I_{V}(0)=I_{V n}(t)-H_{n}(t) .
\end{aligned}
$$

Therefore, we have

$$
\begin{aligned}
\left\|B_{n}(t)\right\|= & \| \frac{2(1-\tau)}{(2-\tau) M(\tau)}\left(\digamma_{1}\left(t, S_{H n}\right)-\digamma_{1}\left(t, S_{H(n-1)}\right)\right)+\frac{2 \tau}{(2-\tau) M(\tau)} \\
& \times \int_{0}^{t}\left(\digamma_{1}\left(y, S_{H n}\right)-\digamma_{1}\left(y, S_{H(n-1)}\right)\right) d y \| \\
\leq & \frac{2(1-\tau)}{(2-\tau) M(\tau)} \|\left(\digamma_{1}\left(t, S_{H n}\right)-\left(\digamma_{1}\left(t, S_{H(n-1)}\right)\right) \|+\frac{2 \tau}{(2-\tau) M(\tau)}\right. \\
& \times \int_{0}^{t}\left\|\left(\digamma_{1}\left(y, S_{H}\right)-\digamma_{1}\left(y, S_{H(n-1)}\right)\right)\right\| d y \\
\leq & \frac{2(1-\tau)}{(2-\tau) M(\tau)} \mu_{1}\left\|S_{H}-S_{H(n-1)}\right\|+\frac{2 \tau}{(2-\tau) M(\tau)} \mu_{1}\left\|S_{H}-S_{H(n-1)}\right\| t .
\end{aligned}
$$

Using the process in a recursive manner gives

$$
\left\|B_{n}(t)\right\| \leq\left(\frac{2(1-\tau)}{(2-\tau) M(\tau)}+\frac{2 \tau}{(2-\tau) M(\tau)} t\right)^{n+1} \mu_{1}^{n+1} a .
$$


Then at $t_{0}$ we have

$$
\left\|B_{n}(t)\right\| \leq\left(\frac{2(1-\tau)}{(2-\tau) M(\tau)}+\frac{2 \tau}{(2-\tau) M(\tau)} t_{0}\right)^{n+1} \mu_{1}^{n+1} a .
$$

By applying the limit on Eq. (31) as $n$ tends to infinity, we get

$$
\left\|B_{n}(t)\right\| \longrightarrow 0
$$

Similarly, we get

$$
\begin{array}{ll}
\left\|C_{n}(t)\right\| \longrightarrow 0, & \left\|D_{n}(t)\right\| \longrightarrow 0, \quad\left\|F_{n}(t)\right\| \longrightarrow 0, \\
\left\|G_{n}(t)\right\| \longrightarrow 0, & \left\|H_{n}(t)\right\| \longrightarrow 0 .
\end{array}
$$

For the uniqueness system (9) solution, we take on contrary that there exists another solution of (9) given by $S_{1 H}(t), E_{1 H}(t), I_{H 1}(t), S_{1 V}(t), E_{1 V}(t)$, and $I_{1 V}(t)$. Then

$$
\begin{aligned}
S_{H}(t)-S_{1 H}(t)= & \frac{2(1-\tau)}{(2-\tau) M(\tau)}\left(\digamma_{1}\left(t, S_{H}\right)-\digamma_{1}\left(t, S_{1 H}\right)\right)+\frac{2 \tau}{(2-\tau) M(\tau)} \\
& \times \int_{0}^{t}\left(\digamma_{1}\left(y, S_{H}\right)-\digamma_{1}\left(y, S_{1 H}\right)\right) d y .
\end{aligned}
$$

Taking norm on Eq. (32), we get

$$
\begin{aligned}
\left\|S_{H}(t)-S_{1 H}(t)\right\| \leq & \frac{2(1-\tau)}{(2-\tau) M(\tau)}\left\|\digamma_{1}\left(t, S_{H}\right)-\digamma_{1}\left(t, S_{1 H}\right)\right\|+\frac{2 \tau}{(2-\tau) M(\tau)} \\
& \times \int_{0}^{t}\left\|\digamma_{1}\left(y, S_{H}\right)-U_{1}\left(y, S_{1 H}\right)\right\| d y .
\end{aligned}
$$

By applying the Lipschitz condition of kernel, we have

$$
\begin{aligned}
\left\|S_{H}(t)-S_{1 H}(t)\right\| \leq & \frac{2(1-\tau)}{(2-\tau) M(\tau)} \mu_{1}\left\|S_{H}(t)-S_{1 H}(t)\right\|+\frac{2 \tau}{(2-\tau) M(\tau)} \\
& \times \int_{0}^{t} \mu_{1} t\left\|S_{H}(t)-S_{1 H}(t)\right\| d y .
\end{aligned}
$$

It gives

$$
\left\|S_{H}(t)-S_{1 H}(t)\right\|\left(1-\frac{2(1-\tau)}{(2-\tau) M(\tau)} \mu_{1}-\frac{2 \tau}{(2-\tau) M(\tau)} \mu_{1} t\right) \leq 0
$$

Theorem 4 The model (9) solution will be unique if

$$
\left(1-\frac{2(1-\tau)}{(2-\tau) M(\tau)} \mu_{1}-\frac{2 \tau}{(2-\tau) M(\tau)} \mu_{1} t\right)>0 .
$$

Proof If condition (36) holds, then (35) implies that

$$
\left\|S_{H}(t)-S_{1 H}(t)\right\|=0
$$


(a)

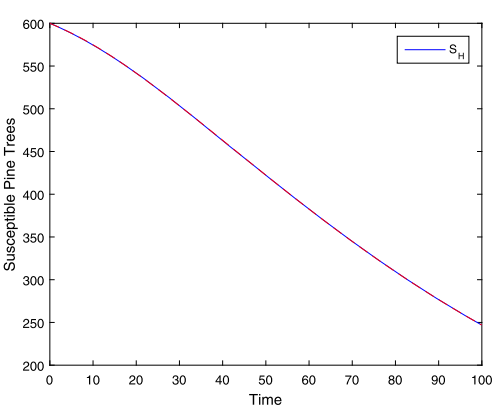

(c)

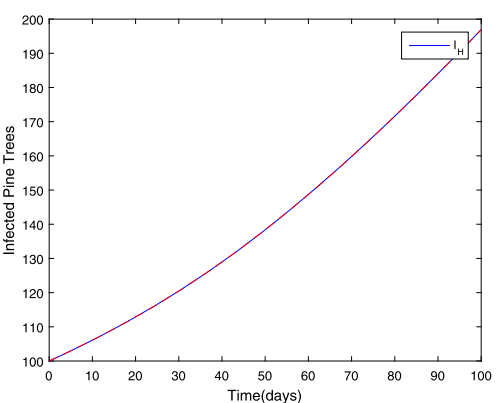

(e)

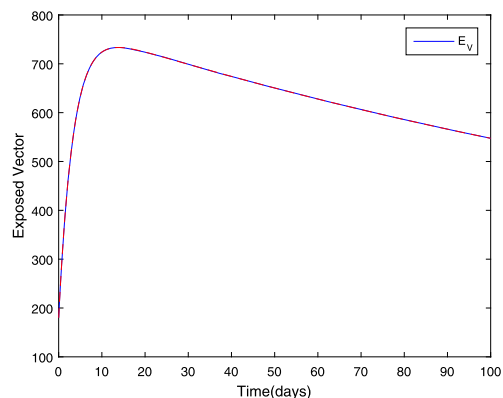

(b)

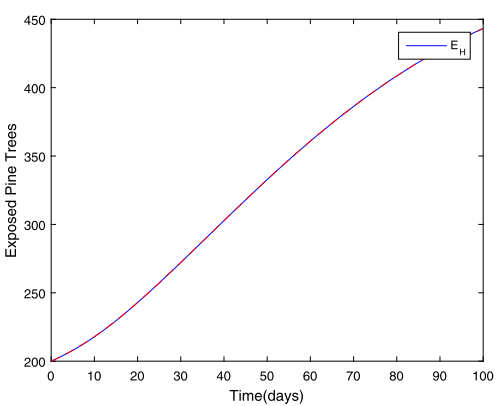

(d)

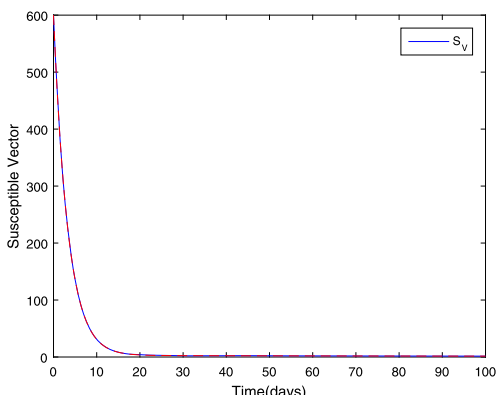

(f)

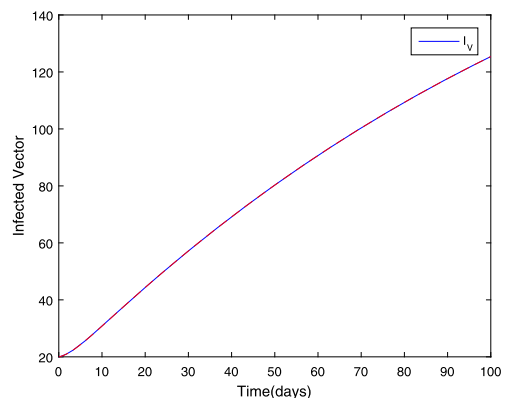

Figure 1 Simulations of pine tree population (a)-(c) and vector population (d)-(f) of the FPWD model (9) for $\tau=1$. (a): Susceptible pine tree; (b): Exposed pine tree; (c): Infected pine tree; (d): Susceptible vector; (e): Exposed vector; (f): Infected vector

Hence, we get

$$
S_{H}(t)=S_{1 H}(t)
$$

On employing the same procedure, we get

$$
\begin{aligned}
& E_{H}(t)=E_{1 H}(t), \\
& I_{H}(t)=I_{1 H}(t), \\
& S_{V}(t)=S_{1 V}(t), \\
& E_{V}(t)=E_{1 V}(t), \\
& I_{V}(t)=I_{1 V}(t) .
\end{aligned}
$$


(a)
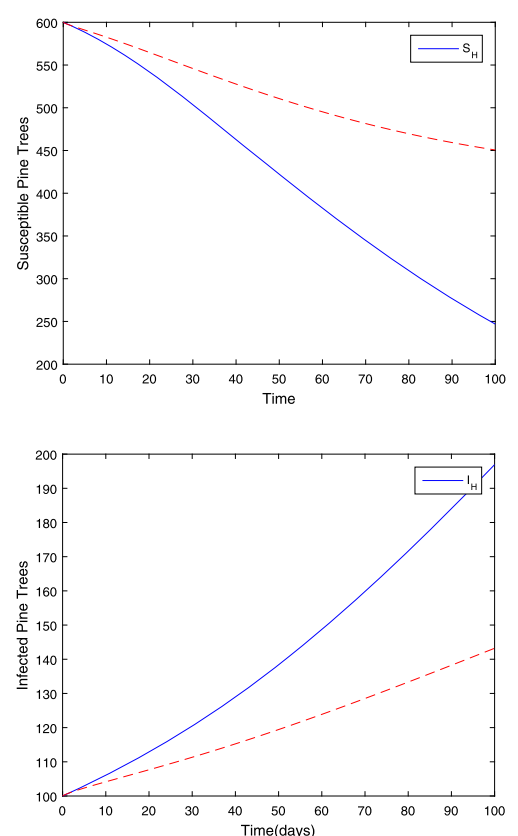

(c)

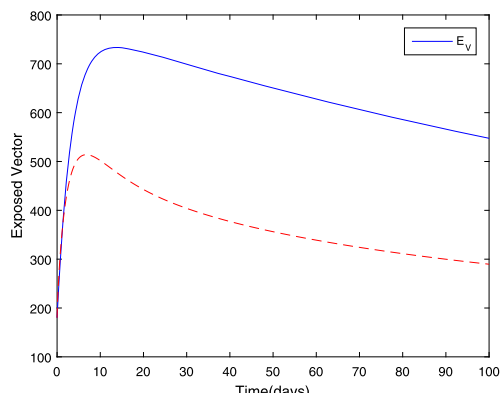

(b)

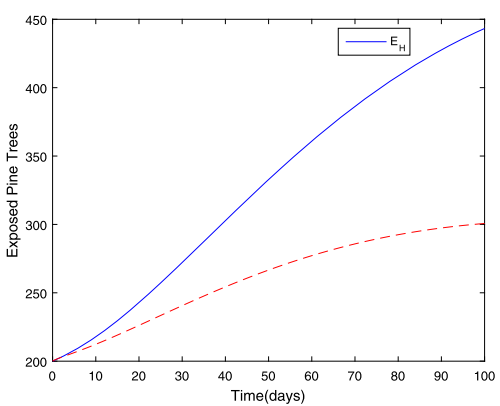

(d)
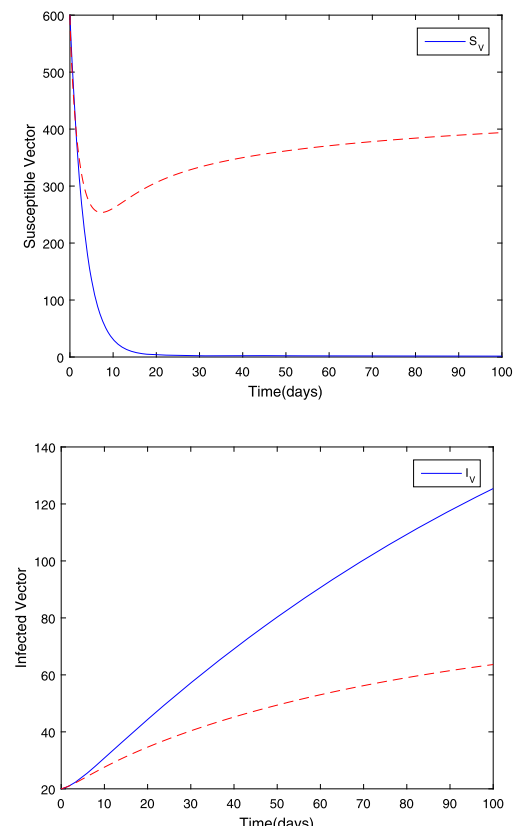

Figure 2 Simulations of pine tree population (a)-(c) and vector population (d)-(f) of the FPWD model (9) for $\tau=0.8$. (a): Susceptible pine tree; (b): Exposed pine tree; (c): Infected pine tree; (d): Susceptible vector; (e): Exposed vector; (f): Infected vector

\section{Numerical results}

The present section aims to find the numerical simulations of the extended fractional order PWD model (9) by variation of the non-integer derivative of order $\tau \in[0,1]$. The values of the parameters used in the numerical simulation are $\Pi_{H}=0.3, K_{1}=0.00022, K_{2}=$ 0.00043, $\theta_{1}=0.01, \gamma_{H}=0.00048, \delta_{H}=0.00317, \psi=0.0000083, \theta_{2}=0.001, \gamma_{V}=0.0028$, $\delta_{V}=0.002, \Pi_{V}=0.8, \beta_{1}=0.003183$. The behavior of the individuals of model (9) for $\tau=1,0.80,0.60,0.40,0.20$ is simulated in Figs. $1-5$, respectively. In each Figure, the behavior of host population (pine trees) is shown by sub-plots (a)-(c), while the graphical behavior of vector population (beetles) is given by sub-plots (d)-(f). Also in each case the solid blue line represents the model simulations plot for $\tau=1$, and the doted red line is the behavior of the model for distinct values of $\tau$ other than 1 . From the numerical results in Figs. 1-5, it is clear that when we decrease the order of fractional derivative $\tau$, the number of both susceptible pine trees and beetles increases, while the exposed and infected pine trees and beetles decrease significantly. From the graphical results we can say 
(a)

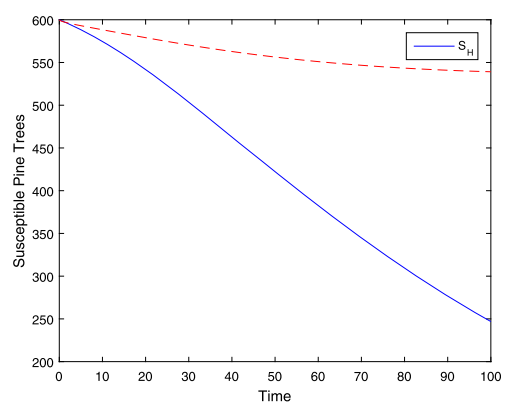

(c)

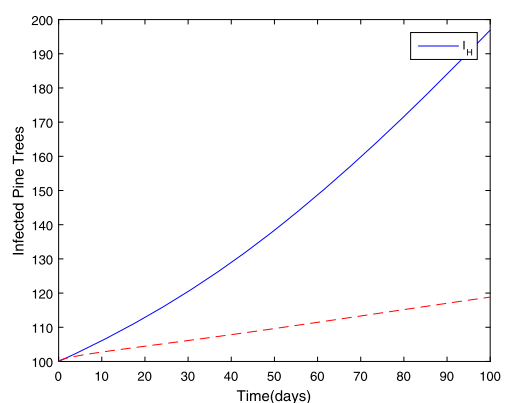

(e)

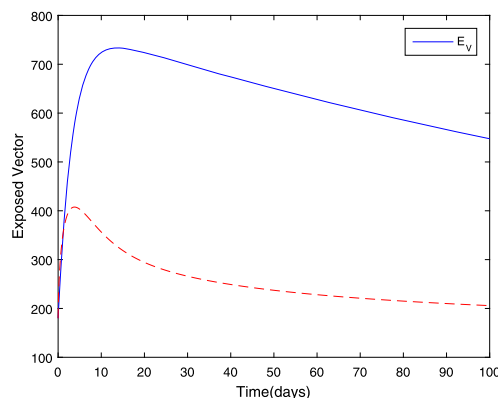

(b)

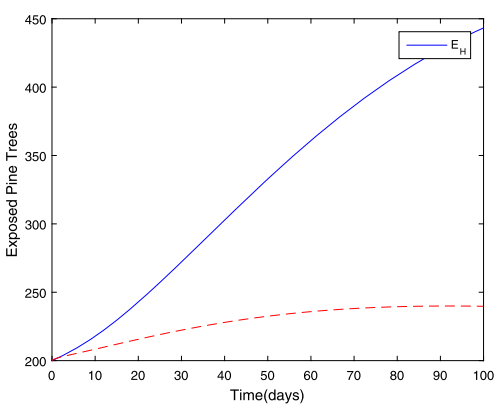

(d)
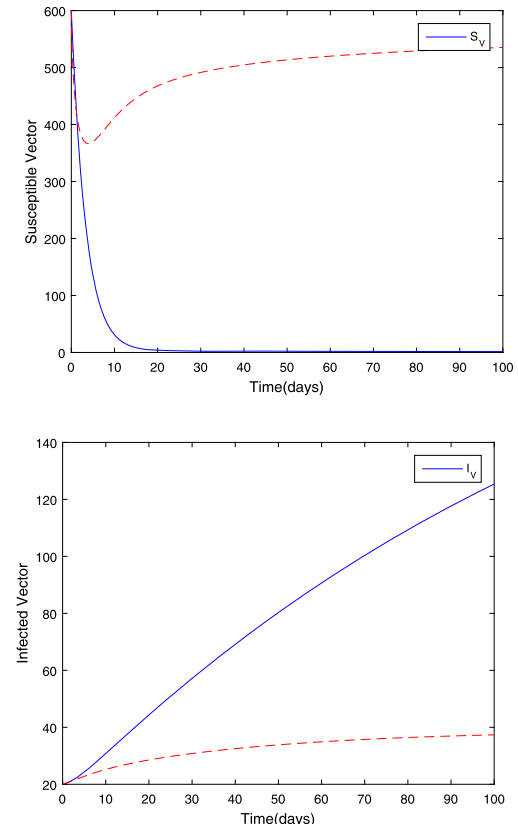

Figure 3 Simulations of pine tree population (a)-(c) and vector population (d)-(f) of the FPWD model (9) for $\tau=0.6$. (a): Susceptible pine tree; $(\mathbf{b})$ : Exposed pine tree; $(\mathbf{c})$ : Infected pine tree; $(\mathbf{d})$ : Susceptible vector; (e): Exposed vector; (f): Infected vector

that the model depends notably on the fractional order derivative, which provides more biologically reasonable results.

\section{Conclusion}

In the present work, we extended the PWD model [12] to fractional order using the Caputo-Fabrizio fractional derivative. The model equilibria and basic reproduction number are explored. The existence and uniqueness of the solution for the FPWD model with $\mathrm{CF}$ derivative are proved in detail. Some numerical simulations are carried out to explore the effect of fractional order. From numerical simulations one can see that when fractional order of derivative $\tau$ decreases, the CF derivative provides more biologically feasible behavior about the dynamic of pine wilt disease. Therefore, we concluded that the newly fractional derivative is very useful for modeling such phenomena. Also, from the graphical behavioral we conclude that the proposed fractional order model provides richer and more flexible results when compared with the corresponding integer order PWD model. 
(a)

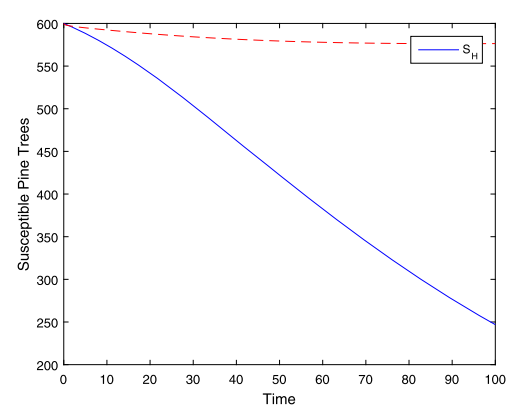

(c)

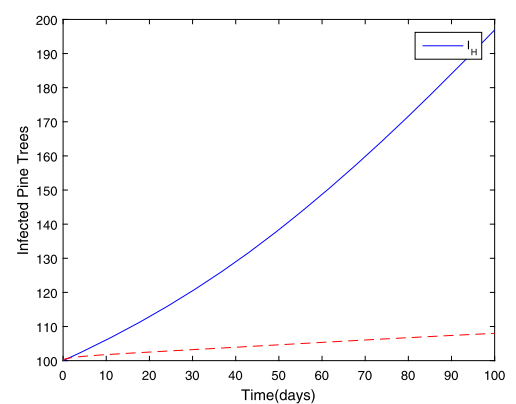

(e)

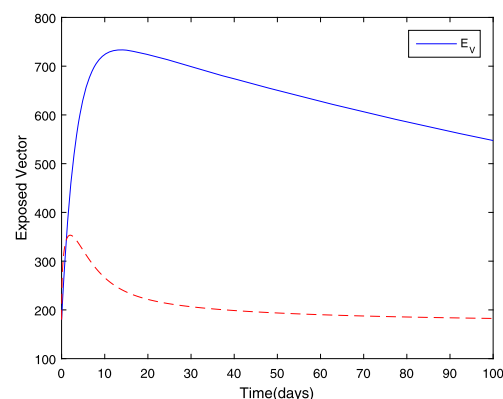

(b)

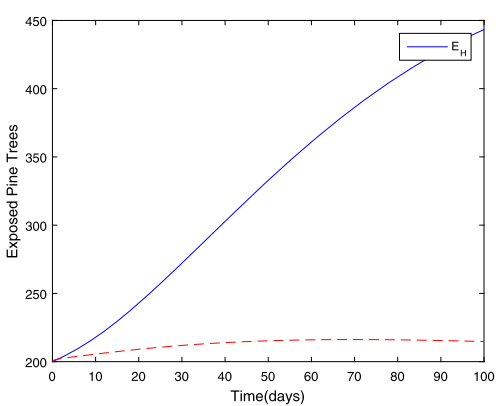

(d)
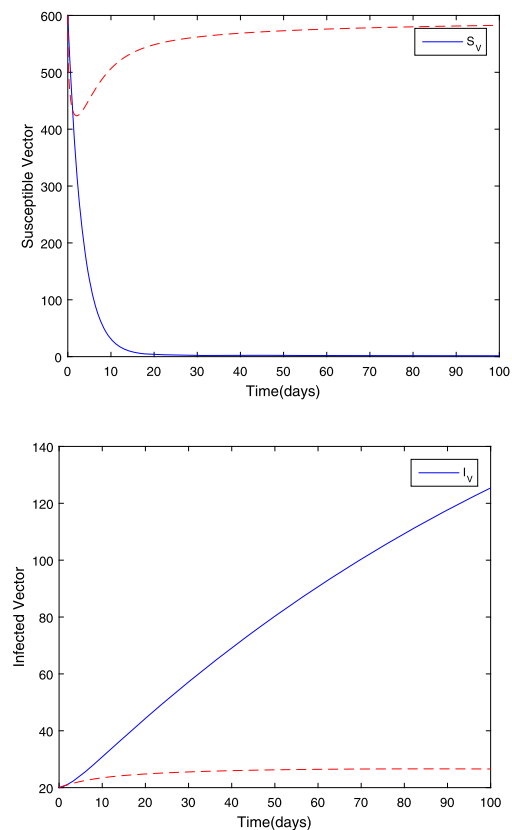

Figure 4 Simulations of pine tree population (a)-(c) and vector population (d)-(f) of the FPWD model (9) for $\tau=0.4$. (a): Susceptible pine tree; $(\mathbf{b})$ : Exposed pine tree; $(\mathbf{c})$ : Infected pine tree; $(\mathbf{d})$ : Susceptible vector; (e): Exposed vector; (f): Infected vector

Thus, we suggest that this new fractional model gives more effective results which could be implemented for the elimination of the pine tree infection.

Acknowledgements

The authors are thankful to the handling editor and respected reviewers for the careful reading and useful comments that greatly improved the presentation of this paper.

Funding

Not applicable.

Availability of data and materials

The data is available without any restrictions.

Competing interests

The authors declare that they have no competing interests.

\section{Authors' contributions}

MAK and KS formulated the fractional order model and obtained the numerical results. SU obtained the existence results for the model. MAK and SU wrote the introduction, abstract, and conclusion. MAK, KOO revised the manuscript. All authors read and approved the final manuscript. 
(a)

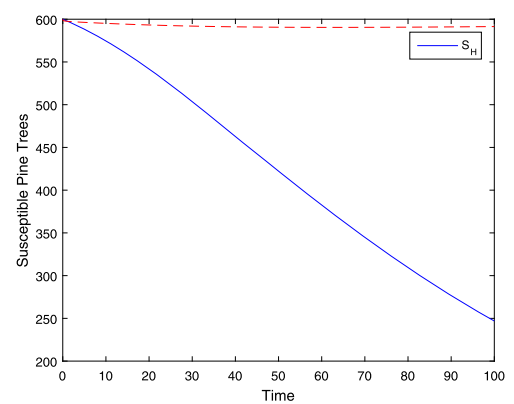

(c)

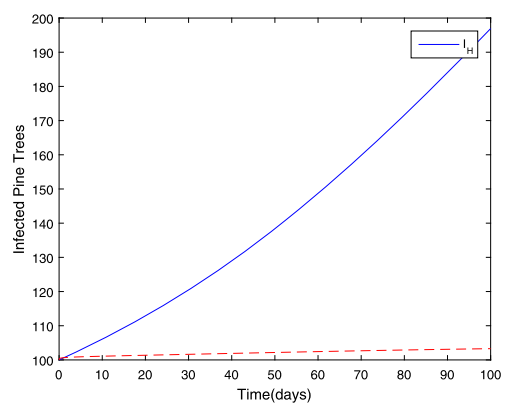

(e)

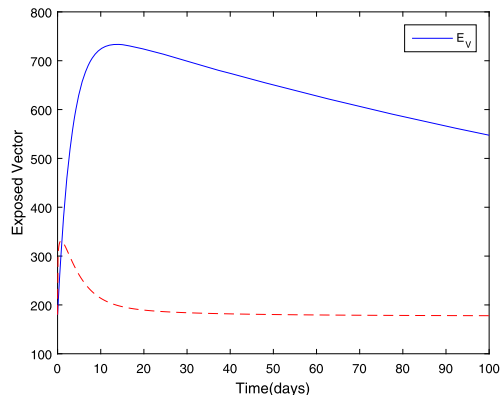

(b)

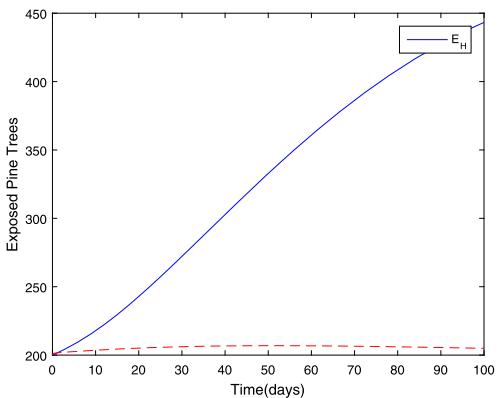

(d)

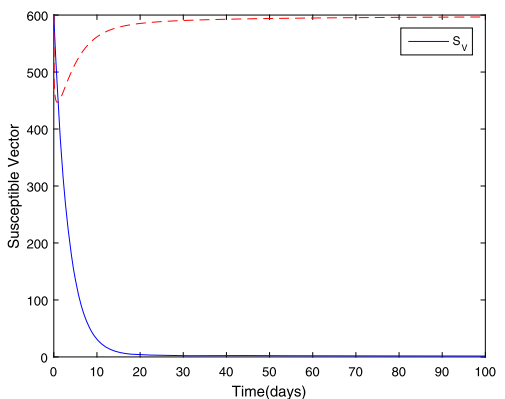

(f)

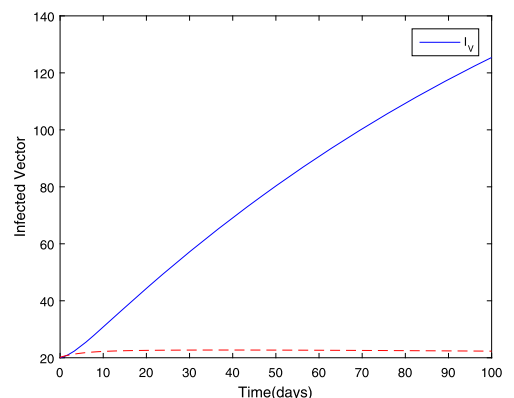

Figure 5 Simulations of pine tree population (a)-(c) and vector population (d)-(f) of the FPWD model (9) for $\tau=0.2$. (a): Susceptible pine tree; (b): Exposed pine tree; (c): Infected pine tree; (d): Susceptible vector; (e): Exposed vector; (f): Infected vector

\section{Author details}

'Department of Mathematics, City University of Science and Information Technology, Peshawar, Pakistan. ${ }^{2}$ Department of Mathematics, University of Peshawar, Peshawar, Pakistan. ${ }^{3}$ Department of Mathematics, Vaal University of Technology, Vanderbij|park, South Africa.

\section{Publisher's Note}

Springer Nature remains neutral with regard to jurisdictional claims in published maps and institutional affiliations.

Received: 22 May 2018 Accepted: 30 October 2018 Published online: 07 November 2018

\section{References}

1. Wang, Y., Cao, J., Li, X., Alsaedi, A.: Edge-based epidemic dynamics with multiple routes of transmission on random networks. Nonlinear Dyn. 91(1), 403-420 (2018)

2. Wang, Y., Jin, Z., Yang, Z., Zhang, Z.-K., Zhou, T., Sun, G.-Q.: Global analysis of an SIS model with an infective vector on complex networks. Nonlinear Anal., Real World Appl. 13(2), 543-557 (2012)

3. Mamiya, Y., Kiyohara, T.: Description of Bursaphelenchus lignicolus n. sp. (Nematoda: Aphelenchoididae) from pine wood and histopathology of nematode-infested trees. Nematologica 18(1), 120-124 (1972)

4. Kiyohara, T., Tokushige, Y.: Inoculation experiments of a nematode, Bursaphelenchus sp., onto pine trees. J. Jpn. For. Soc. 53(7), 210-218 (1971)

5. Mamiya, Y., Enda, N.: Transmission of bursaphelenchus lignicolus (nematoda: Aphelenchoididae) by monochamus alternatus (coleoptera: Cerambycidae). Nematologica 18(2), 159-162 (1972)

6. Morimoto, K., Iwasaki, A.: Role of monochamus alternatus (Coleoptera: Cerambycidae) as a vector of Bursaphelenchus lignicolus (Nematoda: Aphelenchoididae). J. Jpn. For. Soc. 54(6), 177-183 (1972) 
7. Zhao, B.G., Futai, K., Sutherland, J.R., Takeuchi, Y.: Pine Wilt Disease. Springer, Berlin (2008)

8. Rutherford, T., Mamiya, Y., Webster, J.: Nematode-induced pine wilt disease: factors influencing its occurrence and distribution. For. Sci. 36(1), 145-155 (1990)

9. Lee, K.S.: Stability analysis and optimal control strategy for prevention of pine wilt disease. Abstr. Appl. Anal. 2014 Article ID 182680 (2014)

10. Khan, M., Ali, K., Bonyah, E., Okosun, K., Islam, S., Khan, A.: Mathematical modeling and stability analysis of pine wilt disease with optimal control. Sci. Rep. 7(1), 1-19 (2017)

11. Khan, M.A., Khan, R., Khan, Y., Islam, S.: A mathematical analysis of pine wilt disease with variable population size and optimal control strategies. Chaos Solitons Fractals 108, 205-217 (2018)

12. Khan, M.A., Shah, K., Khan, Y., Islam, S.: Mathematical modeling approach to the transmission dynamics of pine wilt disease with saturated incidence rate. Int. J. Biomath. 11(03), 1-20 (2018)

13. Podlubny, I.: Fractional Differential Equations: An Introduction to Fractional Derivatives, Fractional Differential Equations, to Methods of Their Solution and Some of Their Applications (1999)

14. Samko, S.G., Kilbas, A.A., Marichev, O.I., et al.: Fractional Integrals and Derivatives. Theory and Applications. Gordon and Breach, Yverdon (1993)

15. Caputo, M., Fabrizio, M.: A new definition of fractional derivative without singular kernel. Prog. Fract. Differ. Appl. 1(2), $1-13(2015)$

16. Owolabi, K.M., Atangana, A.: Analysis and application of new fractional Adams-Bashforth scheme with Caputo-Fabrizio derivative. Chaos Solitons Fractals 105, 111-119 (2017)

17. Abdulhameed, M., Vieru, D., Roslan, R.: Magnetohydrodynamic electroosmotic flow of Maxwell fluids with Caputo-Fabrizio derivatives through circular tubes. Comput. Math. Appl. 74(10), 2503-2519 (2017)

18. Firoozjaee, M., Jafari, H., Lia, A., Baleanu, D.: Numerical approach of Fokker-Planck equation with Caputo-Fabrizio fractional derivative using Ritz approximation. J. Comput. Appl. Math. 339(10), 367-373 (2018)

19. Abro, K.A., Memon, A.A., Uqaili, M.A.: A comparative mathematical analysis of RL and RC electrical circuits via Atangana-Baleanu and Caputo-Fabrizio fractional derivatives. Eur. Phys. J. Plus 133(3), 113 (2018)

20. Dokuyucu, M.A., Celik, E., Bulut, H., Baskonus, H.M.: Cancer treatment model with the Caputo-Fabrizio fractional derivative. Eur. Phys. J. Plus 133(3), 92 (2018)

21. Ullah, S., Khan, M.A., Faroog, M.: A new fractional model for the dynamics of the hepatitis B virus using the Caputo-Fabrizio derivative. Eur. Phys. J. Plus 133(6), 237 (2018)

22. Diethelm, K:: A fractional calculus based model for the simulation of an outbreak of dengue fever. Nonlinear Dyn 71(4), 613-619 (2013)

23. Area, I., Batarfi, H., Losada, J., Nieto, J.J., Shammakh, W., Torres, Á.: On a fractional order Ebola epidemic model. Adv. Differ. Equ. 2015(1), 278 (2015)

24. Makris, N., Dargush, G., Constantinou, M.: Dynamic analysis of generalized viscoelastic fluids. J. Eng. Mech. 119(8), 1663-1679 (1993)

25. Losada, J., Nieto, J..: Properties of a new fractional derivative without singular kernel. Prog. Fract. Differ. Appl. 1(2), 87-92 (2015)

26. Asif, N., Hammouch, Z., Riaz, M., Bulut, H.: Analytical solution of a Maxwell fluid with slip effects in view of the Caputo-Fabrizio derivative. Eur. Phys. J. Plus 133(7), 272 (2018)

27. Singh, J., Kumar, D., Baleanu, D.: On the analysis of fractional diabetes model with exponential law. Adv. Differ. Equ. 2018(1), 231 (2018)

28. Van den Driessche, P., Watmough, J.: Reproduction numbers and sub-threshold endemic equilibria for compartmental models of disease transmission. Math. Biosci. 180(1-2), 29-48 (2002)

\section{Submit your manuscript to a SpringerOpen ${ }^{\circ}$ journal and benefit from:}

- Convenient online submission

- Rigorous peer review

- Open access: articles freely available online

- High visibility within the field

- Retaining the copyright to your article

Submit your next manuscript at $\gg$ springeropen.com 